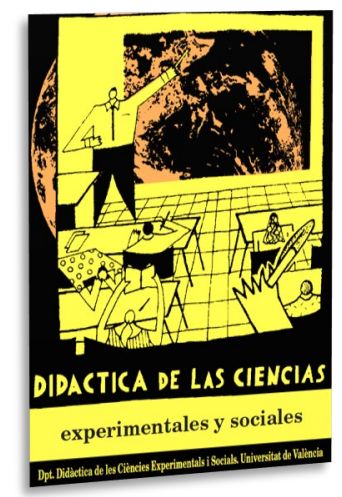

\title{
Conocimiento científico, problemas socioambientales y formación inicial del Profesorado de Primaria
}

\author{
Scientific Knowledge, socioenvironmental \\ problems and Primary School teachers initial \\ training
}

\author{
Lidia López-Lozano \\ Universidad de Sevilla, lidialopez@us.es \\ ORCID iD: https://orcid.org/0000-0002-5181-638X \\ Fátima Rodríguez-Marín \\ Universidad de Sevilla, frodmar@us.es \\ ORCID iD: https://orcid.org/0000-0003-0771-6944 \\ Emilio Solís Ramírez \\ Universidad de Sevilla, esolis@us.es \\ ORCID iD: https://orcid.org/0000-0002-5595-6283
}

\begin{abstract}
RESUMEN: Sin duda, la compleja situación socioambiental actual requiere de una cultura científica que proporcione a la ciudadanía herramientas para intervenir en su realidad. En el contexto de la formación del profesorado, esto toma mayor relevancia si entendemos la educación como motor transformador de una sociedad. Así, presentamos una investigación basada en el diseño y desarrollo de una propuesta didáctica para el aprendizaje de la construcción del conocimiento científico y su construcción social, en el contexto de una problemática socioambiental sobre la que deben emitir un juicio: la ganadería intensiva. El tratamiento multidisciplinar de dicha problemática nos permite, primeramente, presentar la red de problemas y contenido que se han prestado a investigación y, seguidamente, conocer el grado de aprendizaje de los principales rasgos de la metodología investigativa. Se han analizado los 12 proyectos de investigación elaborados por un grupo de estudiantes del Grado en Educación Primaria.
\end{abstract}

Palabras Clave: aprendizaje por investigación, construcción del conocimiento, formación inicial, mapas conceptuales, perspectiva ecosocial

ABSTRACT: Undoubtedly, the current complex socio-environmental situation requires a scientific culture that provides citizens with tools to intervene in their reality. In the context of teacher training, this becomes more relevant if we understand education as the transforming engine of society. To this end, we present a research based on the design and development of a didactic proposal for learning how to construct scientific knowledge and its social construction for a socio-environmental problem on which they must pass judgment: intensive livestock farming. The multidisciplinary treatment of this problem allows us, firstly, to present the network of problems and content that have been subject to research and, subsequently, to know how much the participants learnt about the main features of the research methodology. The 12 research projects, each prepared by a group of students of the Degree of Primary School Education, have been analysed.

KEYWORDS: inquiry-based science education, construction of knowledge, initial training, concept maps, eco-social perspective

Fecha de recepción: marzo de 2021 Fecha de aceptación: julio de 2021 


\section{INTRODUCCIÓN}

En un trabajo anterior (Solís y López-Lozano, 2015) manifestábamos que los estudiantes formaban parte de un mundo complejo con constantes y profundos cambios. Actualmente, pensamos que esas circunstancias no han cambiado sustancialmente e incluso puede que se hayan agudizado. Por ello, en el momento presente, los estudiantes de las diversas titulaciones de Ciencias de la Educación viven en contextos similares. Asimismo, entendemos que en el papel de futuros educadores tienen una profunda responsabilidad, ya que la escuela debe asumir el reto de promover el acercamiento a la cultura y al conocimiento científico. En definitiva, deberán acercar a su futuro alumnado a la educación científica, de manera que les proporcionen herramientas para intervenir en su mundo o por lo menos cuestionar su entorno (Vilches y Gil-Pérez, 2012). Como indica Pujol (2003, p.13):

Educar a los escolares a ser ciudadanos y ciudadanas de un mundo más justo y más sostenible es algo a lo que la educación científica no puede renunciar. Abrir caminos al profesorado para que les enseñen a aprender que la realidad del mundo puede verse desde muchas perspectivas, entre ellas la de la ciencia, y el interés que tiene utilizar sus "gafas" para mirar la realidad.

Qué duda cabe, que algunos de los cambios que, lamentablemente, se encuentran dentro de la más rabiosa actualidad, son los que se están produciendo por efecto del cambio climático (COP25, 2019). Para abordar estos cambios con una visión global y crítica, el conocimiento científico se perfila como un medio riguroso y válido en el desarrollo intelectual de las personas (Rivero y Solís, 2008). Presentamos aquí un trabajo llevado a cabo con futuros docentes de Primaria para aproximarlos al proceso de construcción del conocimiento científico, abordando dicha construcción desde una perspectiva social y ambiental, lo que podríamos denominar una perspectiva ecosocial.

Estamos convencidos de la idoneidad de trabajar partiendo de problemas (García, Porlán y Navarro, 2017; García-Díaz, 2001) y de lo que entendemos por problemas socioambientales (Moreno y Moreno, 2015). Coincidimos con Moreno y Moreno (2015), que/cuando exponen que no hay problemática ambiental sin dimensión social. En este sentido, hemos propuesto una actividad que aproxime a los estudiantes a un proceso de investigación, que les permita la construcción del conocimiento científico a través del planteamiento de un problema relacionado con la educación ambiental (García-Díaz, 2001). Esta propuesta permite un doble propósito formativo: de un lado, ponerles en contacto con el proceso investigativo y de construcción del conocimiento científico y, de otro lado, abordar el carácter social de la ciencia, esto es, los aspectos éticos, morales e incluso afectivos relacionados con la reflexión y la toma responsable de decisiones (España y Prieto, 2010; Rivero y Solís, 2008).

En este último sentido, el de la toma responsable de decisiones, ya en 1985, The Royal Society de Londres, elaboró un documento, The public understanding of science (también denominado informe Bodmer), en cuyo Prefacio, se expresa (p. 5):

Más que nunca, la gente necesita entender la Ciencia si tiene que verse sometida a procesos de toma de decisiones a niveles nacional o local, en la dirección de industrias, en empleos que requieren semi o total especialización, en el ejercicio del voto como ciudadanos o en la toma de una serie de decisiones personales. Al publicar este informe, la Royal Society espera señalar esta necesidad para una toma de conciencia general de la naturaleza de la ciencia y muy especialmente del modo como la Ciencia y la Tecnología impregnan nuestra sociedad...

Por otra parte, no es nuevo en la literatura relacionada con la enseñanza de las ciencias (Aragón, 2017; García Barros, 2016; García-Carmona, Vázquez y Manassero, 2012; Manassero y Vázquez Alonso, 2017; Lederman y Lederman, 2014; Rivero y Wamba, 2011), que el "cómo" conciba el profesorado los aspectos relacionados con la ciencia, su naturaleza, su desarrollo, la construcción del 
conocimiento científico, etc., son de especial trascendencia en cómo desarrollará dicho profesorado sus intervenciones en el aula. Es decir, qué visión intentará trasmitir a su alumnado sobre estas cuestiones y, por ende, que formación ciudadana se puede ir construyendo en los mismos, respecto al análisis que puedan realizar ante los presentes y futuros problemas de la sociedad, sobre todo, aquellos que tengan implicaciones de carácter científico, así como las posibles soluciones que desde distintos estamentos se pueden proponer. Pensamos que igual de importante que este análisis es la actitud que pueden adaptar ante determinados tópicos que suelen acompañar a esta problemática, como es la presencia de la pseudociencia, o ante la idea tan extendida de que la naturaleza es sabia y el problema será absorbido y/o reconducido por la misma, precisamente, para situarse en una postura negacionista respecto a la existencia de los problemas ambientales.

Respecto a los aspectos que caracterizan el trabajo de problemas socioambientales en el aula, Moreno y Moreno (2015) destacan: el potencial que esta manera de trabajar tiene como generador de conocimientos; la posibilidad de analizar la problemática desde distintos enfoques; su virtualidad para acceder a cuestiones complejas, que de otro modo serían difíciles de abordar; la necesidad de poner en juego tanto el conocimiento científico como el cotidiano, que son problemas que, sin duda, pueden afectar a la vida presente o futura de las personas y que, finalmente, permiten que el sistema educativo se convierta en elemento que propicie una reflexión crítica sobre dichos problemas.

Como indicábamos anteriormente, la propuesta que presentamos y analizamos intenta, por una parte, aunar la idea de construcción del conocimiento científico y la aplicación de dicho conocimiento al trabajo con problemas ambientales (Barraza y Castaño, 2012). Y, de otra, llevar a cabo un proceso formativo con los estudiantes del Grado de Educación Primaria. Para ello, consideramos necesario situarnos en una perspectiva de modelo de enseñanza que integre una determinada concepción del conocimiento científico y de la construcción del mismo, con una propuesta metodológica que se base en la investigación escolar.

En este sentido, no todos los modelos de enseñanza posibles son adecuados para conjugar los aspectos antes citados. Así, proponemos un sistema de enseñanza que, respecto a la concepción sobre la ciencia, su construcción y su enseñanza, se mueva entre el evolucionismo y un relativismo moderado. Es decir, la ciencia como construcción humana, que cambia y evoluciona en función de múltiples dimensiones (Ziman 1986): dimensión psicológica, epistemológica, sociológica, tecnológica y ética y socio-política. A esto deberíamos añadir la contribución que realiza Morin (1995) con el paradigma de la complejidad que, en el contexto en el que estamos desarrollando este trabajo, plantea que el sistema escolar sea complejo y cambiante.

Para ello habrá que intentar desarrollar una idea de un conocimiento científico que tenga en cuenta que la Ciencia es un conjunto organizado de conocimientos, válido para explicar la realidad y útil para facilitarnos la vida. Es decir, un conocimiento que es fundamentado y no anecdótico, que es demostrable, ya sea empírica o lógicamente, que tiene poder predictivo, que es intersubjetivo y no es neutro (Rivero y Wamba, 2011). No obstante, como indican Escrivá-Colomar y Rivero-García (2017), esta no es la concepción mayoritaria entre el profesorado sobre la ciencia y su naturaleza, sino más bien, aquella más cercana a una posición tradicional y relacionada con el positivismo y con un idealismo basado en la infalibilidad y el absolutismo de los procesos científicos.

Por otra parte, el modelo de enseñanza que puede integrar una concepción del conocimiento científico y una metodología basada en la investigación escolar, de acuerdo con la tipificación de García (2000), atendiendo, fundamentalmente a categorías relacionadas con elementos curriculares (qué y cómo enseñar y evaluar) y epistemológicos (naturaleza e historia de la ciencia, génesis del conocimiento científico, objetividad de la investigación científica, la ciencia y el conocimiento de la realidad socioambiental), es el denominado Modelo basado en la Investigación Escolar (MIE). En una revisión realizada por Solís, Porlán, Rivero y Martín del Pozo (2012, p. 497), en referencia a los contenidos en este tipo de Modelo de enseñanza, se apunta: "Conocimiento escolar que integra saberes (disciplinares, cotidianos, ambientales,...). Visión relativa, evolutiva e integradora". Asimismo, en relación con la metodología, se indica (Solís et al., 2012, p. 498): 
Metodología basada en la investigación del alumnado. Se trabaja en torno a problemas. La secuencia de las actividades viene determinada por el propio problema. El estudiante construye y reelabora su conocimiento mediante procesos de negociación en el aula. El profesorado como coordinador y/o facilitador de los procesos de investigación que se dan en el aula (tanto del alumnado como del propio proceso).

Como indican, además, Rivero, Martín del Pozo, Solís y Porlán (2017), se ha generalizado en la literatura internacional el término: enseñanza de las ciencias basada en la indagación o IBSE (Inquiry-based Science Education) (Harlen 2015), para referirse a este modelo, coincidente en gran medida con el Modelo de Investigación Escolar, de forma que:

Enseñar y aprender por investigación es un proceso complejo que requiere que el profesorado desarrolle un conocimiento práctico, rico y profundo, tanto epistemológico (en relación con la naturaleza de la ciencia y del conocimiento), como científico (en relación al contenido que se esté tratando y su desarrollo histórico) y didáctico (relacionado con las dificultades que pueden presentar los estudiantes, las actividades más relevantes para provocar contrastes significativos, etc.) (Rivero et al., 2017, p. 108).

Finalmente, pensamos que la idea de la construcción social del conocimiento científico es determinante para la práctica docente mediante la investigación de problemas, el trabajo en equipo y la objetividad social. Debe estar presente en la formación inicial del profesorado y, de acuerdo con García-Carmona, Vázquez y Manassero (2011), se deben intensificar la alfabetización científica, tanto en el currículo de ciencia escolar como en la formación inicial de profesorado.

\section{MÉTODO}

\subsection{Objetivos}

Se llevó a cabo una experiencia formativa que permitiese al futuro profesorado abordar las cuestiones anteriormente planteadas acerca del conocimiento científico, contextualizado, además, sobre una problemática real y relacionada con un problema socioambiental que consideramos relevante, durante la cual pudiesen desarrollar habilidades investigativas y actitud crítica y reflexiva, trabajar en equipo y comunicar sus experiencias y decisiones.

Por ello, el propósito de este trabajo es describir una estrategia útil en la formación del profesorado que se concreta en la problemática de la ganadería intensiva con potencial para trabajar un amplio abanico de cuestiones socioambientales interrelacionadas. Desde un planteamiento que nos permite pensar en clave de sistema (Caselles 2008) y, por tanto, de las relaciones de sus elementos: la ganadería intensiva es el todo y los elementos, o partes, las problemáticas investigadas que pueden ir desde modelos agroalimentarios, a cuestiones climáticas pasando por hábitos de consumo, salud, modelo socioeconómico,... lo que permitiría además asumir el principio de emergencia de forma que el todo sea más que la suma de las partes. A su vez, su desarrollo nos permite conocer el grado de aprendizaje de los principales rasgos de la metodología investigativa.

\subsection{Diseño de la propuesta y contexto}

El cómo abordar las ideas antes expuestas se puede hacer de variadas formas. Bien mediante lecturas, documentos de contraste y posteriores puestas en común, o bien mediante la participación en proyectos de investigación escolar que, aunque con las limitaciones inherentes al ámbito en el que se desarrolla, no tienen por qué adolecer de: planteamiento de hipótesis o preguntas, contener cierto trabajo de campo, ser riguroso, consultar fuentes y tener posibilidad de comunicar sus conclusiones 
en el contexto en el que se desarrolla. Esta segunda opción es la escogida ya que pensamos que tanto conceptual como metodológicamente es más rica y permite, además, incorporar el trabajo cooperativo, trabajar con más diversidad de elementos a la hora del contraste e incluso una cierta aproximación a los juegos de simulación como recurso para el análisis de sistemas complejos e interrelacionados (Ballenilla, 1989; Manassero Mas y Vázquez Alonso, 2017; Mengascini y Menegaz, 2005) y como elemento de debate y reflexión en la formación inicial del profesorado (Solís, Luna y Soler, 1999; Vázquez, Jiménez y Mellado, 2007).

Por otra parte, el trabajo de investigación se puede proponer partiendo de un problema de libre elección, en cuyo caso se usa fundamentalmente los puntos tres y siguientes del guion que aparece en la figura 1, dando lugar a propuestas del tipo: investigación de las vitaminas y los aditivos en los alimentos, la dependencia de la sociedad actual respecto a la telefonía móvil... como ha ocurrido en otros trabajos de aula. También se puede realizar a partir de una temática concreta como, por ejemplo, la energía (Solís y López-Lozano, 2015), las disoluciones (Kraser y Hernández, 2020) o planteando un problema socioambiental común (Brocos y Jiménez Aleixandre, 2020), como es nuestro caso. Cada una de las opciones presenta cualidades diferentes en cuanto a la riqueza de conocimientos que se pueden dar, el fomento de la creatividad de cada grupo o el hecho de permitir una mayor profundización en el problema y una mayor relevancia de las complejidades e interrelaciones que el problema pueda presentar.

La estrategia formativa se desarrolló con un grupo de 49 estudiantes de $2^{\circ}$ curso del Grado de Educación Primaria de la Escuela Universitaria de Osuna (centro oficial adscrito a la Universidad de Sevilla) en la asignatura anual y obligatoria de Didáctica de Ciencias Experimentales, en el curso 2018-19. Tratándose de una actividad de carácter grupal se conformaron 12 grupos de 3-5 componentes.

La actividad se enmarca en la primera parte del primer bloque de la asignatura (que trata la materia objeto de enseñanza: la Ciencia) dedicado a la Naturaleza de la Ciencia y el conocimiento científico. Este bloque se articula en torno a cuatro problemas clave interrelacionados: qué se entiende por ciencia, cómo se construye, cómo cambia y la ciencia como actividad humana (López-Lozano, 2020). Para acercarnos más adecuadamente a la idea de cómo se construye el conocimiento científico, los métodos a través de los cuales se desarrolla y, también, los dilemas éticos frente a una situación problemática o con cierta controversia, se les propone realizar un trabajo de investigación, teniendo en cuenta las siguientes características (Rivero et al., 2017):

a) Debe tratarse de cuestiones desconocidas, de ahí el interés por investigarlas.

b) Deben ser cuestiones que se puedan resolver, con realismo, en un tiempo breve (en este caso se estima alrededor de dos semanas).

c) Dicha investigación no debe hacerse solo mediante consulta bibliográfica, sino, también, mediante observaciones empíricas o trabajo de campo, directamente sobre los aspectos del problema investigado.

Bajo estos supuestos, se les plantea una hipotética situación sobre una problemática real, de la actualidad y próxima a su entorno por las características del municipio del centro universitario y, también, residencia de muchos de ellos. Esto se expone en la Figura 1 junto al procedimiento a seguir que hizo las veces de guion en la realización de la actividad y posterior informe de trabajo que se entrega y se analiza. 
FiguRA 1. Guion de trabajo del Proyecto de Investigación

La propuesta de investigación concierne a la siguiente situación:

Una multinacional agrícola trae a nuestro municipio de la comarca sevillana un proyecto de instalación de una macrogranja de porcino o vacuno, esto queda aún por decidir. Desde el ayuntamiento, nos plantean investigar la idoneidad de dicha propuesta y nuestro posicionamiento personal frente a esta posibilidad.

Para ello, el esquema de trabajo (Informe a elaborar) que se seguiría en el desarrollo de esta actividad es el siguiente:

1. Ideas iniciales: cada equipo planteará los aspectos, cuestiones o problemas a investigar que creen que hay alrededor de esta situación. Esto se puede esquematizar a modo de mapa conceptual de manera que podemos distinguir tanto las relaciones que puedan existir entre las diversas cuestiones planteadas como el contenido que se abordaría, de modo que nos pueda ofrecer una visión global de la situación-problema.

(esquema o mapa conceptual inicial)

2. Puesta en común de lo anterior por todos los equipos y elaboración final y grupal de un mapa conceptual con los diferentes problemas. Tras esto, cada equipo o grupo de equipos abordará una de las cuestiones-problema surgidas. En el caso de que algunos equipos aborden el mismo problema se hará desde perspectivas distintas complementarias.

(esquema o mapa conceptual final)

Cada equipo inicia su investigación, para ello se puede seguir los siguientes pasos (puntos 3 al 7, Cañal y Rivero, 1999):

3. Define el problema (o los problemas) que va a investigar.

4. La(s) hipótesis inicial(es) que se tiene respecto a cada problema (se trata de expresar las suposiciones, intuiciones, dudas, creencias, etc., que tenemos en el momento inicial).

5. Planificación de la investigación (fases del trabajo, fuentes de información que vamos a consultar, elaboración de los instrumentos que necesitemos para obtener resultados, etc.).

6. Descripción de cada paso o actividad realizada en el proceso de investigación (¿cómo se ha hecho y qué información ha aportado?).

7. Conclusiones obtenidas, tanto respecto a los resultados propiamente dichos de la investigación como respecto al proceso de trabajo que hayamos seguido. Las conclusiones relativas a los resultados deben presentarse sintéticamente en relación al problema y hacer alusión a la(s) hipótesis que teníamos (la amplía, la contradice, la refuerza...). Respecto al proceso, se puede aludir a qué actividades han salido bien, cuáles no, qué problemas se han presentado, que modificaciones introducirías si tuviésemos que repetir la investigación, etc.; así como al grado de validez del proceso en general para aprender.

8. Finalmente, se debe tomar una decisión apoyándoos en vuestras conclusiones:

\section{¿Apoyáis la instalación o no? ¿Por qué?}

9. Presentación y comunicación de conclusiones al resto de los equipos.

10. Tras conocer las conclusiones alcanzadas por todos los grupos, ¿mantenéis la postura declarada anteriormente o consideráis modificarla? ¿Por qué?

Fuente: elaboración propia

Se parte del mapa o esquema conceptual inicial que cada grupo elabora con los aspectos que creen que se han de tener en cuenta en torno al problema planteado. Esto actúa como actividad de exploración de sus ideas iniciales. Tras una puesta en común se acuerda un mapa final de contenido a partir del cual cada equipo elige su problema de investigación. La siguiente fase es de desarrollo e intergrupal que conduce a la toma de decisión, se exponen las conclusiones obtenidas por todos y se vuelve a replantear la decisión tomada.

Resaltamos la intencionalidad de los últimos puntos (8-10) con el objetivo de superar los obstáculos que se presentan en formación inicial en la construcción del conocimiento científico y su impacto social (Acevedo et al., 2005; García-Carmona et al., 2011; López-Lozano, 2020). De un lado, la visión parcialmente contextualizada de la ciencia cuando analizamos un problema solo desde una 
perspectiva, o teniendo en cuenta una o pocas variables, a una visión contextualizada con aportaciones de diversas fuentes y considerando un amplio espectro de variables nos puede llevar a una mejor toma de decisión. De otro lado, por el paralelismo con el conocimiento científico como producto de trabajo colectivo frente a la visión individualista de la ciencia.

FIGURA 2. Instrumento de análisis de las investigaciones escolares

\begin{tabular}{|c|c|c|c|}
\hline \multicolumn{4}{|c|}{$\begin{array}{l}\text { 1. DIMENSIÓN METODOLÓGICA } \\
\text { Diseño y aplicación de procesos experimentales: ¿Cómo desarrollan la parte de la } \\
\text { construcción del conocimiento científico? }\end{array}$} \\
\hline \multirow[b]{2}{*}{ 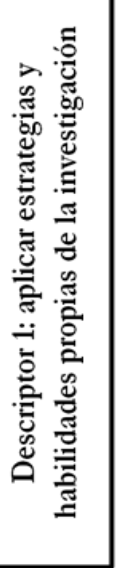 } & Nivel inicial N1 & Nivel inter medio $\mathbf{N 2}$ & Nivel avanzado $\mathrm{N} 3$ \\
\hline & $\begin{array}{l}\text { Formula una hipótesis } \\
\text { (H, en adelante) que no } \\
\text { se puede comprobar o } \\
\text { no es relevante o no } \\
\text { responde al Problema } \\
\text { planteado, no propone } \\
\text { un diseño experimental. } \\
\text { Los datos recogidos no } \\
\text { responden a la } \\
\text { problemática y no se } \\
\text { identifican o argumenta } \\
\text { posibles } \\
\text { errores/confinsiones }\end{array}$ & $\begin{array}{l}\text { Plantea la } \mathrm{H} \text { aunque } \\
\text { no identifica las } \\
\text { variables que } \\
\text { intervienen. El diseño } \\
\text { responde parcialmente } \\
\text { a su propósito } \\
\text { resultando una } \\
\text { información con baja } \\
\text { relevancia. Registra los } \\
\text { datos aunque } \\
\text { imprecisa y } \\
\text { desorganizadamente }\end{array}$ & $\begin{array}{l}\text { Identifica el problema y } \\
\text { formula la H que encaja } \\
\text { con dicho problema } \\
\text { teniendo en cuenta el } \\
\text { marco y la relación entre } \\
\text { variables. Planifica un } \\
\text { diseño experimental } \\
\text { coherente con la H, para } \\
\text { ello selecciona y analiza } \\
\text { la información relevante. } \\
\text { Registra los datos, } \\
\text { resultados adecuados. }\end{array}$ \\
\hline \multicolumn{4}{|c|}{ 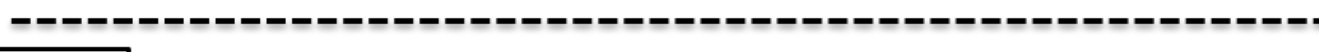 } \\
\hline 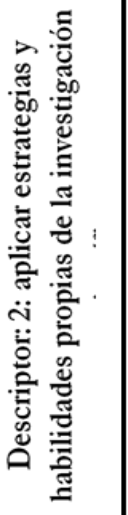 & $\begin{array}{l}\text { No se plantea si los } \\
\text { resultados son } \\
\text { fiables o no } \\
\text { No reconoce las } \\
\text { limitaciones del } \\
\text { estudio (relaciones } \\
\text { con otros } \\
\text { parámetros) } \\
\text { Expone la decisión, } \\
\text { pero no la } \\
\text { fundamenta }\end{array}$ & $\begin{array}{l}\text { Identifica las variables, } \\
\text { pero no sus relaciones. } \\
\text { Confusión entre } \\
\text { observación e inferencia. } \\
\text { Intenta fundamentar los } \\
\text { resultados, aunque no sea } \\
\text { adecuado. } \\
\text { Indeterminación respecto } \\
\text { a si necesitaría más } \\
\text { pruebas. Reconoce } \\
\text { limitaciones, pero no las } \\
\text { identifica. Expone la } \\
\text { decisión }\end{array}$ & $\begin{array}{l}\text { Identifica patrones o } \\
\text { variables que influyen } \\
\text { en el fenómeno y sus } \\
\text { relaciones. Reconoce si } \\
\text { los resultados } \\
\text { confirman o no la Hi. } \\
\text { Reconoce las } \\
\text { limitaciones del trabajo } \\
\text { (puede proponer otros } \\
\text { interrogantes) } \\
\text { Argumenta, en función } \\
\text { a su búsqueda, la } \\
\text { decisión tomada }\end{array}$ \\
\hline \multicolumn{4}{|c|}{$\begin{array}{l}\text { 2. DIMENSIÓN ACTITUDINAL } \\
\text { Adopta actitudes cientificas: } ¿ \text { Cuál es la postura ante el problema? }{ }^{\text {EEn qué basan sus }} \\
\text { decisiones? ¿Cambian? }\end{array}$} \\
\hline 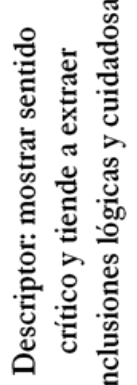 & $\begin{array}{l}\text { N1: Le cuesta } \\
\text { diferenciar entre } \\
\text { ciencia y creencia. } \\
\text { No relaciona su } \\
\text { indagación con su } \\
\text { decisión }\end{array}$ & $\begin{array}{l}\text { N2: Puede mostrar duda, } \\
\text { principalmente, basada en } \\
\text { el procedimiento seguido } \\
\text { en la investigación. Puede } \\
\text { presentar cuestionamiento. } \\
\text { Prevalece la descripción } \\
\text { resumida de lo obtenido } \\
\text { frente a la interpretación. } \\
\text { Prevalece el conocimiento } \\
\text { parcial. }\end{array}$ & $\begin{array}{l}\text { N3: Muestra sentido } \\
\text { crítico/duda, diferencia } \\
\text { las creencias y opiniones } \\
\text { del conocimiento } \\
\text { científico apoyando sus } \\
\text { argumentaciones en lo } \\
\text { hallado. Presenta } \\
\text { cuestionamiento tras } \\
\text { tener un conocimiento } \\
\text { más amplio }\end{array}$ \\
\hline
\end{tabular}

Fuente: adaptado de Pigrau, T. y Sanmartí, N. (2013). La competencia científica. Rúbrica. En Tresor de recursos. Evaluar para aprender. Disponible en: https://www.tresorderecursos.com/copia-de-2-rubrica-comp-cc 


\subsection{Instrumento y análisis de la información}

Para el análisis, contamos con 12 informes de trabajo elaborados por los grupos siguiendo el guion dado para la actividad (ver figura 1). Desde un enfoque descriptivo cualitativo (Creswell, 2013), se analiza su contenido de manera que podamos establecer resultados según dos dimensiones atendiendo a la finalidad de dicha actividad: construcción del conocimiento científico y construcción social del mismo.

Para ello, usamos como instrumento de análisis un sistema de categoría y niveles (LópezLozano, 2020; Rodríguez-Marín, Fernández y García, 2014) (figura 2) adaptado de la rúbrica para evaluar la competencia científica de Pigrau y Sanmartí (2013). La dimensión metodológica nos informa de la idoneidad del proceso de investigación según el planteamiento de los pasos seguidos en la investigación (definir el problema, emisión de hipótesis, coherencia entre éstas y la planificación de la investigación) y la fundamentación de las conclusiones alcanzadas. La dimensión actitudinal nos permite conocer la postura adoptada ante el problema, su argumentación y posición final tras la puesta colectiva.

\section{DesarRollo del TRABAJO Y RESUltados}

\subsection{Primera fase: construcción del mapa de contenido}

El primer paso es la preparación, organización y presentación del contenido. Esto implica un trabajo de documentación previo sobre la problemática socioambiental y el ajuste del conocimiento y/o intereses del que aprende. Asimismo, según la finalidad del trabajo de comprender y actuar en la realidad, optamos por la problematización del contenido y proponemos organizarlo y presentarlo bajo un mapa de contenidos-problemas (García-Díaz et al., 2017).

El proceso seguido es mediante reelaboración con el alumnado, de manera que se parte de sus ideas sobre las macrogranjas a nivel intergrupal y sin intervención. Esta primera propuesta de contenido o problemáticas a tratar queda recogida a modo de esquema-mapa en los trabajos del alumnado (correspondiente al paso 1 del guion, en figura 1) (ver ejemplos en la figura 3), los cuales se han analizado siguiendo la rúbrica diseñada por Morón Monge, Morón Monge y Abril López (2020).

FIGURA 3. Ejemplos de esquemas y mapas iniciales

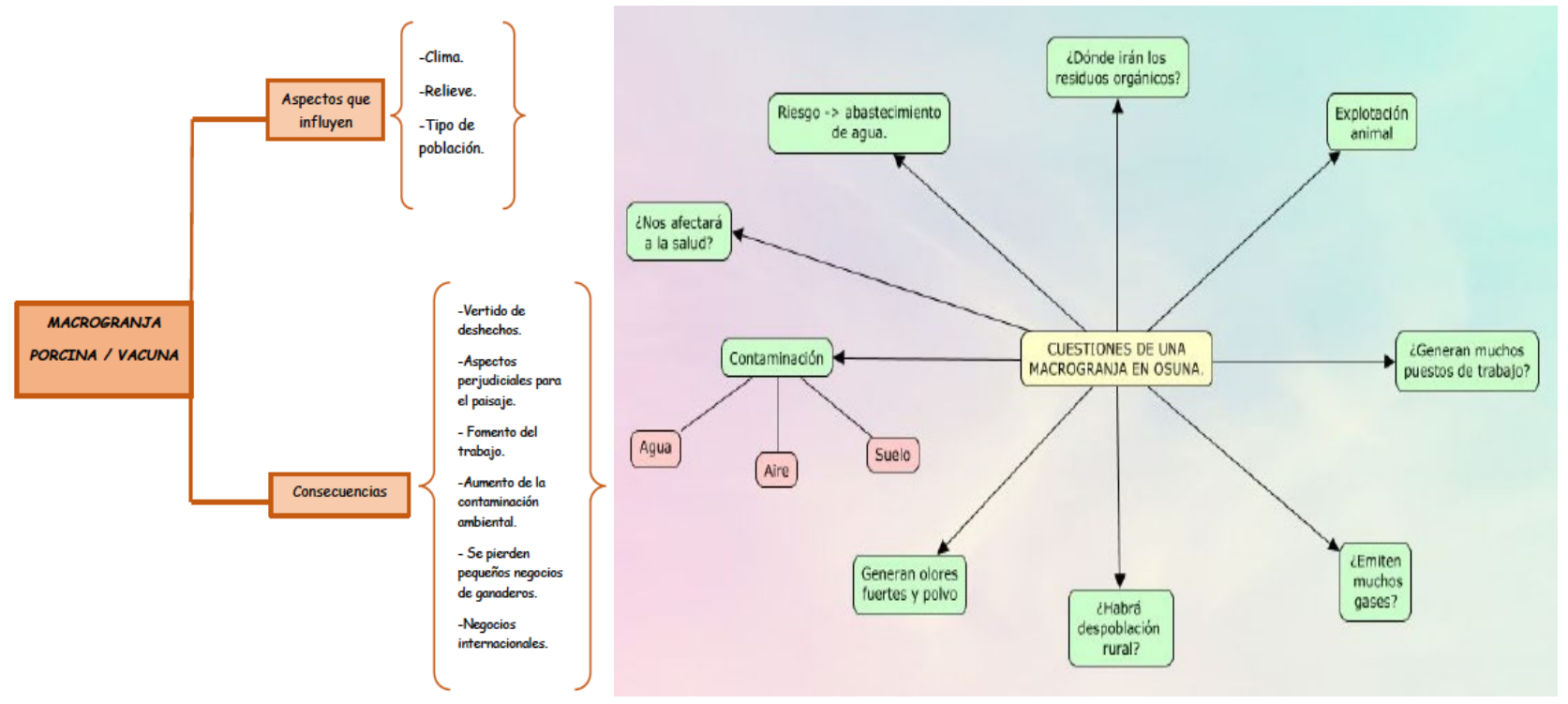

Fuente: Grupo 6 (a) y Grupo 7 (b). 
Los mapas que presenta el alumnado al inicio en esta fase se encuentran en el nivel I, siendo como un esquema, con un planteamiento simple, donde los nexos que se emplean son una traslación de la información de partida, sin análisis ni interpretación (Morón Monge et al., 2020).

A partir de aquí, en el aula mediante la puesta en común de cada propuesta y con la guía de la docente (primera autora del manuscrito) se termina conformando un mapa de contenido-problemas asociados a la situación que ayuda a los equipos a elegir la cuestión que les interesa investigar (correspondiente al paso 2 del guion, en figura 1). En este momento, el alumnado representa los contenidos de forma que se podría categorizar en el nivel II del trabajo antes citado (figura 4). Aparecen las diversas perspectivas desde las que se puede abordar la problemática -perspectiva biológica, socioambiental y socioeconómica-. Esto les ayuda a establecer mejores relaciones entre el contenido, a entender la complejidad del asunto que, a su vez, conlleva comprender que las respuestas a un mismo problema van a depender de la perspectiva y los valores que se adopten (Bravo Torija y Jiménez Aleixandre, 2013).

FIGURA 4. Ejemplo mapa post-puesta en común

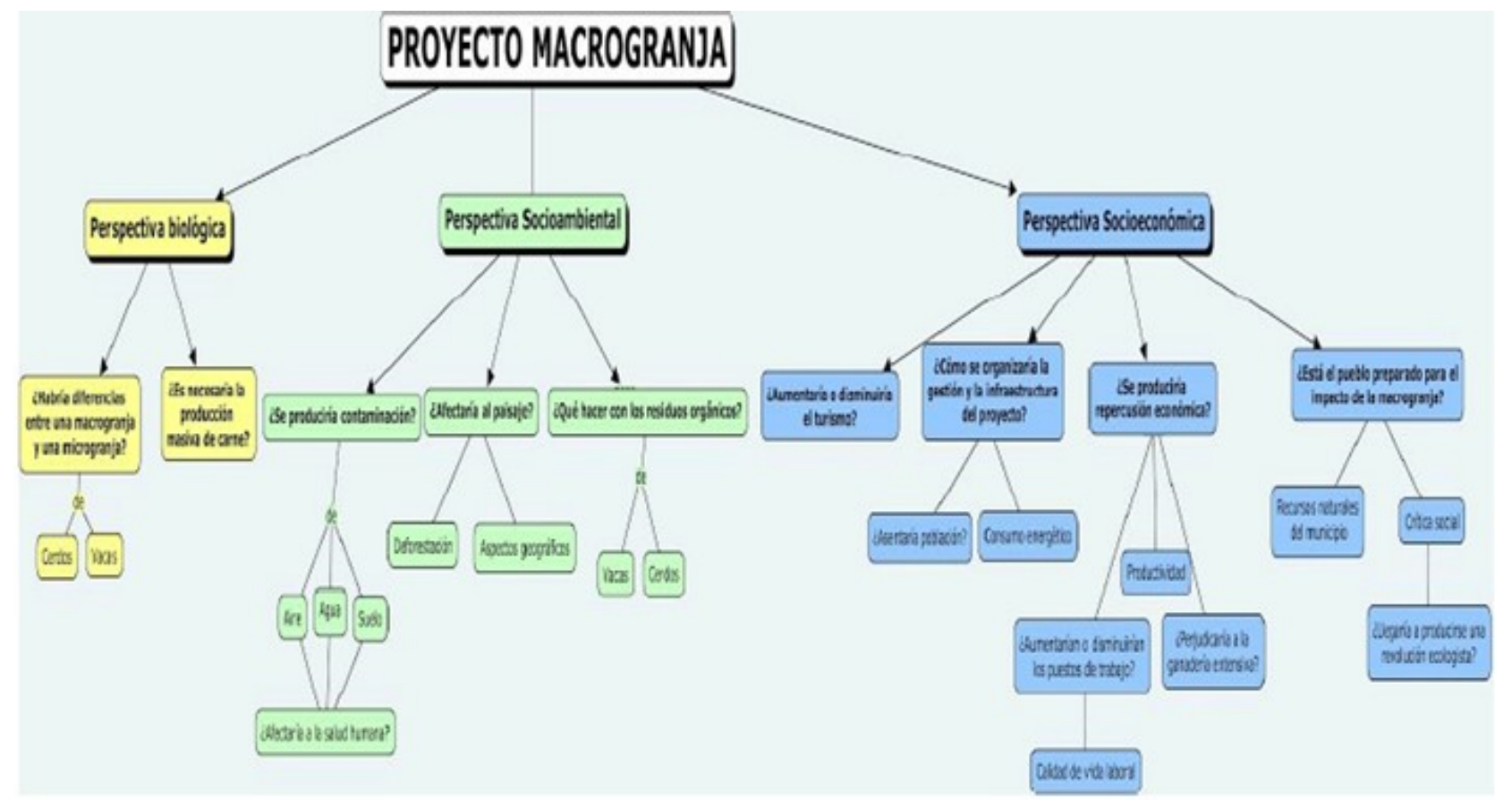

Fuente: Grupo 2

Y, por último, se presenta un mapa de contenido-problema que pueda servir para futuros trabajos (figura 5), en el que se intenta reflejar la complejidad de la problemática socioambiental planteada, se observan las perspectivas, las temáticas claves, los problemas planteados en esta investigación, así como otros que se pueden plantear y las interrelaciones entre los mismos. Dicho mapa se elabora por los profesores autores del presente trabajo a partir de las propuestas de problemas de los estudiantes. 
FIGURA 5. Mapa contenido-problema final guía del trabajo de investigación vinculado a las Macrogranjas

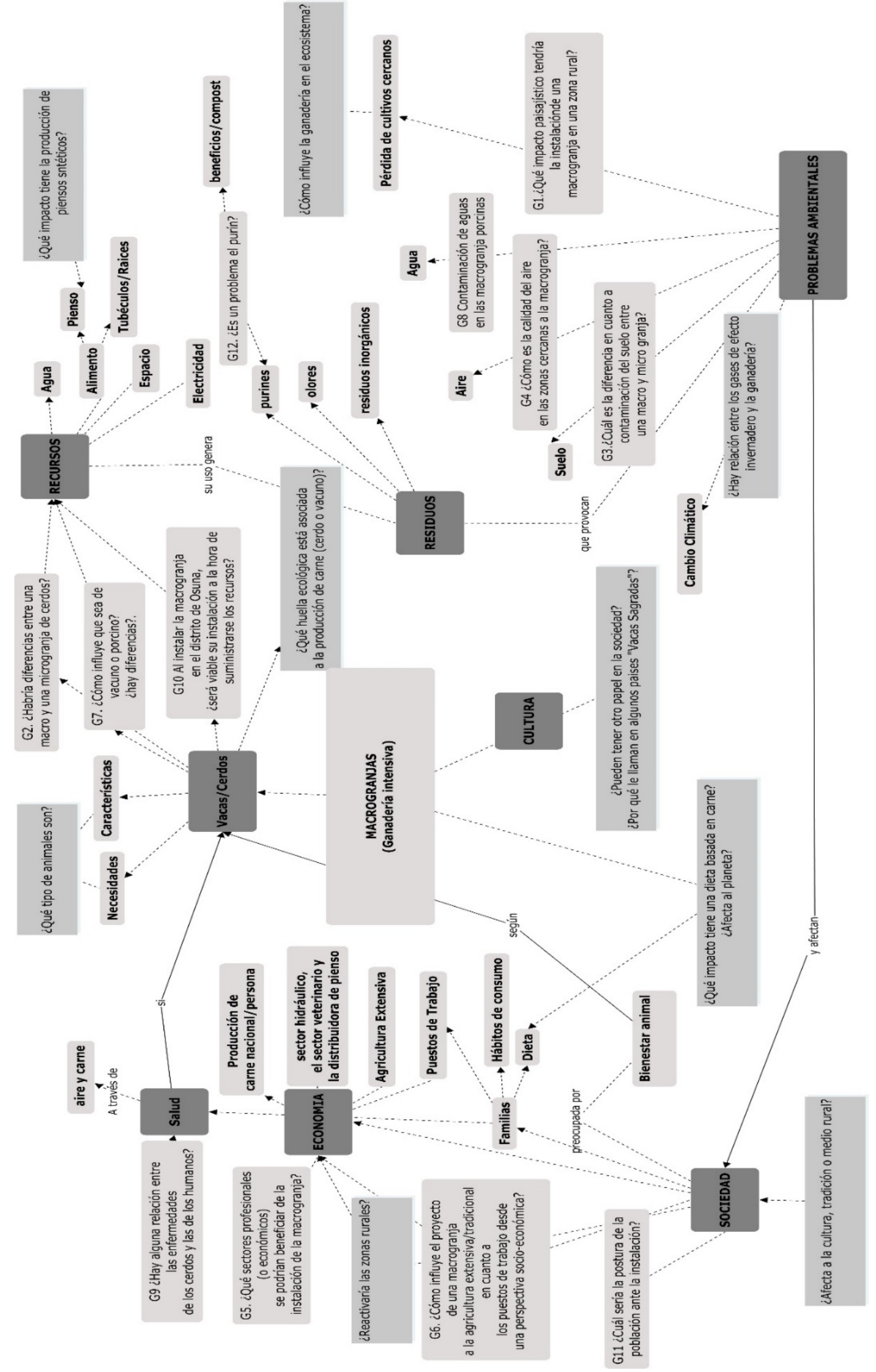

Fuente: elaboración propia 


\subsection{Segunda fase: investigación y toma de decisiones}

Basándose en los contenidos identificados que esbozan la situación problema en el mapa conceptual, los grupos investigaron acerca de los temas que se exponen en la tabla 1. Además, en dicha tabla, se resume el nivel de aprendizaje alcanzado según los niveles establecidos en cada dimensión y descriptor (D1 y D2, respectivamente).

TABLA 1. Problemáticas que se investigan y su tratamiento.

\begin{tabular}{|c|c|c|}
\hline PROBLEMA PLANTEADO & $\begin{array}{c}\text { Dimensión } 1 \\
\text {-Método seguido- }\end{array}$ & $\begin{array}{c}\text { Dimensión } 2 \\
\text {-Decisión- }\end{array}$ \\
\hline \multirow{2}{*}{$\begin{array}{l}\text { G1 ¿Qué impacto paisajístico tendría } \\
\text { la instalación de una macrogranja en } \\
\text { una zona rural? }\end{array}$} & $D_{1}: N 3 / D_{2}: N 3$ & $\begin{array}{l}\text { A favor, con } \\
\text { condiciones }\end{array}$ \\
\hline & $\begin{array}{l}\text { Documentación/trabajo de campo/ } \\
\text { Entrevista a agricultor }\end{array}$ & $\begin{array}{c}\mathrm{N} 2 \\
\text { Cambian }\end{array}$ \\
\hline \multirow[t]{2}{*}{$\begin{array}{l}\text { G2 ¿Habría diferencias entre una } \\
\text { macro y una microgranja de cerdos? }\end{array}$} & $\mathrm{D}_{1}: \mathrm{N} 2 / \mathrm{D}_{2}: \mathrm{N} 2$ & $\begin{array}{l}\text { En contra } \\
\qquad \mathrm{N} 2\end{array}$ \\
\hline & $\begin{array}{l}\text { Entrevista a ganaderos y consulta } \\
\text { bibliográfica }\end{array}$ & \\
\hline \multirow{2}{*}{$\begin{array}{l}\text { G3 ¿Cuál es la diferencia en cuanto a } \\
\text { contaminación del suelo entre una } \\
\text { macro y micro granja? }\end{array}$} & $\mathrm{D}_{1}: \mathrm{N} 3 / \mathrm{D}_{2}: \mathrm{N} 3$ & $\begin{array}{l}\text { En contra } \\
\quad \mathrm{N} 3\end{array}$ \\
\hline & $\begin{array}{l}\text { Experimento físico-químico (presencia } \\
\text { de materia orgánica y } \mathrm{Ph} \text { ) y entrevista a } \\
\text { ganaderos }\end{array}$ & \\
\hline \multirow[t]{2}{*}{$\begin{array}{l}\text { G4 ¿Cómo es la calidad del aire en las } \\
\text { zonas cercanas a la macrogranja? }\end{array}$} & $\mathrm{D}_{1}: \mathrm{N} 1 / \mathrm{D}_{2}: \mathrm{N} 1$ & $\begin{array}{l}\text { En contra } \\
\qquad \mathrm{N} 1\end{array}$ \\
\hline & Consultas bibliográficas & \\
\hline \multirow{2}{*}{$\begin{array}{l}\text { G5 ¿Qué sectores profesionales (o } \\
\text { económicos) se podrían beneficiar de } \\
\text { la instalación de la macrogranja? }\end{array}$} & $\mathrm{D}_{1}: \mathrm{N} 3 / \mathrm{D}_{2}: \mathrm{N} 3$ & $\begin{array}{l}\text { En contra } \\
\qquad \mathrm{N} 3\end{array}$ \\
\hline & $\begin{array}{l}\text { Trabajo de búsqueda de información y } \\
\text { entrevista a estudiantes de Ingeniería } \\
\text { agronómica y de CCAA }\end{array}$ & \\
\hline \multirow{2}{*}{$\begin{array}{l}\text { G6 ¿Cómo influye el proyecto de una } \\
\text { macrogranaja a la agricultura } \\
\text { extensiva o tradicional en cuanto a } \\
\text { puestos de trabajo desde una } \\
\text { perspectiva socioeconómica? }\end{array}$} & $D_{1}: N 3 / D_{2}: N 3$ & $\begin{array}{c}\text { A favor } \\
\text { parcialmente }\end{array}$ \\
\hline & $\begin{array}{l}\text { Consulta bibliográficas y entrevista a un } \\
\text { trabajador de explotación ganadera }\end{array}$ & $\begin{array}{l}\text { N3 } \\
\text { Cambian }\end{array}$ \\
\hline \multirow{2}{*}{$\begin{array}{l}\text { G7 ¿Cómo influye que sea de vacuno } \\
\text { o porcino? ¿Hay diferencias? }\end{array}$} & $\mathrm{D}_{1}: \mathrm{N} 1 / \mathrm{D}_{2}: \mathrm{N} 1$ & En contra \\
\hline & $\begin{array}{l}\text { Entrevista a ganadero y consultas } \\
\text { bibliográficas }\end{array}$ & $\mathrm{N} 2$ \\
\hline \multirow[t]{2}{*}{$\begin{array}{l}\text { G8 Contaminación de aguas en las } \\
\text { macrogranja porcinas }\end{array}$} & $\mathrm{D}_{1}: \mathrm{N} 3 / \mathrm{D}_{2}: \mathrm{N} 2$ & $\begin{array}{l}\text { En contra } \\
\qquad \mathrm{N} 3\end{array}$ \\
\hline & Documentación bibliográfica & \\
\hline $\begin{array}{l}\text { G9 ¿Hay alguna relación entre las } \\
\text { enfermedades de los cerdos y las de } \\
\text { los humanos? }\end{array}$ & $\mathrm{D}_{1}: \mathrm{N} 3 / \mathrm{D}_{2}: \mathrm{N} 3$ & $\begin{array}{l}\text { En contra } \\
\qquad \mathrm{N} 3\end{array}$ \\
\hline
\end{tabular}




\begin{tabular}{|c|c|c|}
\hline & Documentación/ entrevista a ganadero & \\
\hline $\begin{array}{l}\text { G10 Al instalar la macrogranja en el } \\
\text { distrito de Osuna, ¿será viable su } \\
\text { instalación a la hora de suministrarse } \\
\text { los recursos? }\end{array}$ & $\begin{array}{l}D_{1}: N 3 / D_{2}: N 3 \\
\text { Información en el Ayuntamiento/ } \\
\text { consulta bibliográfica }\end{array}$ & $\begin{array}{c}\text { En contra } \\
\text { N3 }\end{array}$ \\
\hline $\begin{array}{l}\text { G11 ¿Cuál sería la postura de la } \\
\text { población ante la instalación? }\end{array}$ & $\begin{array}{l}\mathrm{D}_{1}: \mathrm{N} 2 / \mathrm{D}_{2}: \mathrm{N} 2 \\
\text { Encuesta poblacional }\end{array}$ & $\begin{array}{c}\text { En contra } \\
\text { N3 }\end{array}$ \\
\hline G12. ¿Es un problema el purín? & $\begin{array}{l}\mathrm{D}_{1}: \mathrm{N} 2 \text { / } \mathrm{D}_{2}: \mathrm{N} 3 \\
\text { Documentación bibliográfica }\end{array}$ & $\begin{array}{l}\text { A favor } \\
\text { N2 } \\
\text { Cambian }\end{array}$ \\
\hline
\end{tabular}

Fuente: elaboración propia

Todos los equipos mostraron capacidad para identificar problemas viables para su investigación en las condiciones dadas. Principalmente, encontramos trabajos que aplican adecuadamente las estrategias y habilidades propias de la investigación alcanzando conclusiones de manera fundamentada (N3 en ambos descriptores). Ejemplos son el G5 y G6 que establecen variables teniendo en cuenta sus relaciones y dependencias. El G10, acota los recursos a terreno construible, pienso, agua y electricidad:

P: "Al instalar la macrogranja en el distrito de Osuna, ¿será viable su instalación a la hora de suministrarse de recursos?". En esos recursos incluimos terreno construible, pienso, agua y electricidad, entre otros.

H: Viendo las ideas que se han manejado en la puesta en común inicial y después de plantearnos nuestro problema a investigar, suponemos que la macrogranja podría instalarse sin problema en el municipio puesto que no habría problema para encontrar un terreno donde construirla y, por tanto, el suministro tanto de agua como de electricidad no sería un problema. A parte del suministro de pienso que, observando que es un pueblo mayormente de profesión agrícola, no tendríamos problema alguno (sic) (G10).

Siguiendo con ejemplos, el G3, sobre contaminación del suelo, se planteó averiguar el nivel de materia orgánica y la acidez $(\mathrm{pH})$ como variables para conocer las características del suelo. Encontraron alternativas ante las limitaciones que se les planteaba - no tenían acceso a una macrogranja, decidieron comparar una granja convencional con un suelo sin pasto de ganado y poder establecer así una aproximación; también, averiguaron sobre la gestión de los excrementos de las reses entrevistando a ganadero y de sus respuestas extrapolaron al caso de la ganadería intensiva-.

Por lo que, para alcanzar conclusiones, identifican, de alguna manera, variables que influyen en el fenómeno y sus relaciones, se reconoce si los resultados confirman o no la $\mathrm{H}$ y las limitaciones propias del trabajo. La decisión tomada se basa en dichos datos, léase, por ejemplo:

Observamos que nuestro grupo control (la muestra del suelo de cultivo) no ha funcionado realmente como grupo control. Desconocíamos que ese suelo estaría tratado con fertilizantes orgánicos y sintéticos y eso ha hecho que los resultados que hemos obtenido no sean del todo los esperados.

A pesar de eso y centrándonos en los resultados obtenidos en los dos tipos de muestras del interior la granja (el suelo en el que las vacas están habitualmente y el suelo en el que pastan de forma eventual) podemos concluir que aceptamos nuestras hipótesis iniciales,(...) Si volviéramos a 
realizar nuestra investigación tendríamos que seleccionar mejor el terreno en el que no pasta ganado y asegurarnos de que fuera un terreno en el que no se vertieran fertilizantes ni residuos orgánicos para que pudiese funcionar verdaderamente como grupo control. En vista de los resultados obtenidos y apoyándonos en las respuestas del ganadero a nuestras preguntas, no apostaríamos por la construcción de la macrogranja. Creemos que se produciría una importante contaminación del suelo y las aguas no sólo porque los excesos de excrementos de las vacas los contaminen de forma natural sino porque, además, pensamos que no hay demasiado control en cuanto a la gestión de los mismos. (sic)(G3).

No solo exponen las limitaciones o dificultades enfrentadas, sino que plantean posibilidades de ampliar el estudio, de realizar modificaciones, por ejemplo:

el problema que se nos ha presentado es no tener cerca una macrogranja ni información objetiva cercana (...) Si tuviésemos que repetir la investigación nuestra hipótesis inicial cambiaría (...) en cuanto a la perspectiva socio-económica y quizás la centraríamos en conocer si la macrogranja afectaría al empleo pero no solo de la ganadería extensiva sino también relacionada con otros ámbitos. (sic) G6.

Por otro lado, el nivel intermedio, N2, representa, básicamente, un planteamiento en el que no se identifican las variables que intervienen. Como puede ser el G2, ante la posibilidad o no de que se den diferencias entre una macro y una micro granja, la $\mathrm{H}$ es simplemente que sí, sin definir parámetros para establecer ni la comparativa ni justificar dicha formulación. No obstante, en el diseño se van estableciendo (como es el caso de la alimentación del animal, consumo de agua, la producción y consumo cárnico, el espacio disponible para el animal, factores ambientales y salud e higiene), pero se registran los datos de manera desorganizada y sin relación, consecuencia de lo primero.

En cuanto a las conclusiones, en este nivel de aprendizaje, la tendencia es más descriptiva que interpretativa, a modo de resumen de lo obtenido, basando su toma de decisión en una ponderación de las variables identificadas sin considerar sus relaciones, léase:

Teniendo en cuenta nuestra investigación, concluimos que tiene dos aspectos a favor que serían la rentabilidad y la creación de puestos de trabajo, pero la rentabilidad sería exclusivamente para el propietario de la empresa y se crearían muy pocos puestos de trabajo. Por eso, no apoyamos la instalación, ya que pensamos que no es necesaria la sobreproducción de carne y no estamos de acuerdo con las condiciones de espacio en las que viven los animales, son seres vivos que deberían vivir en su entorno natural en la medida de lo posible (sic) (G2).

No se dan trabajos íntegramente en el nivel inicial, N1, sino que presentan algunos aspectos que lo caracterizan, sobre todo, en el desarrollo metodológico. En estos casos, la dificultad recae en la confusión en la formulación de problemas y de hipótesis, léase el planteamiento de una de las problemáticas formuladas por el G4 "La contaminación del aire por la gran cantidad de cerdos, y lo que ello conlleva, que hay en una macrogranja". La naturaleza de la $\mathrm{H}$ hace que sea inviable comprobarla, no es relevante o bien no responde al problema planteado. Así, el diseño metodológico, puede parecer desconectado, no atiende a los planteamientos iniciales. Las conclusiones siguen esta línea procedimental quedando poco fundamentadas, no se plantean si lo hallado es fiable, es decir, no se reconocen las limitaciones: "Mediante algunas entrevistas hemos podido constatar nuestra hipótesis inicial, en la que afirmamos que afecta de manera negativa la presencia de la macrogranja" (sic) (G4).

Respecto a la dimensión actitudinal, desde la visión parcial fruto de la indagación de su problemática, la mayoría (9) concluye posicionándose contrarios a la instalación. Tras conocer todas las investigaciones (visión más completa del problema), éstos se reafirman y, los 3 restantes a favor, modifican su decisión, finalmente, contrarios a la instalación. 
En cuanto al nivel argumentativo, muestran actitud crítica, reconociendo la parcialidad y fragmentación de la información, así como la relación entre variables. Por ejemplo, el G10 que, a pesar de comprobar la viabilidad de la instalación, son contrarios teniendo en cuenta los datos obtenidos de consumo (luz, agua, alimentación...) y las implicaciones sociales vinculadas al empleo y los factores ambientales. El grupo que se planteaba la posible relación entre enfermedades del animal y el humano (G9), basa su posicionamiento estableciendo relaciones más ricas (cuestiones de producción y consumo justo). Léase, este grupo y otro a favor, como ejemplos:

No la apoyamos, ya que la salud de los cerdos va a estar más en peligro. Si hubiera más granjas pequeñas que puedan servir sus productos de calidad a un precio digno para los productores $\mathrm{y}$ consumidores, no se generarían los problemas e incertidumbres de las macrogranjas (sic) (G9).

Sí, desde la perspectiva socio-económica hemos llegado a la conclusión que la instalación de la macrogranja puede generar nuevos puestos de trabajo sin afectar a los puestos ya existentes de la agricultura extensiva. No obstante, habría que saber si a largo plazo, esto podría cambiar y cómo afectaría a otros tipos de empleos en la comarca para que fuera positivo para todos (sic) (G6).

No obstante, algunas decisiones se ciñen exclusivamente a lo investigado, a veces, apostillando condiciones ideales, resultando poco fundamentada en lo obtenido:

En el caso que las partes se comprometan y hagan lo necesario para realizar lo expuesto anteriormente, en lo que a nuestro problema se refiere no habría ninguna objeción a la construcción de la macro granja, verificado anteriormente que la zona tiene suficiente agua para todo su abastecimiento. Pues en estas circunstancias, no habría pérdida de cultivo en la zona, sino todo lo contrario ayudaría en beneficio del mismo (sic) (G1).

\section{CONCLUSIONES}

El estudio que presentamos pretende compartir una estrategia formativa en el contexto de la formación del profesorado válida para promover prácticas científicas indagatoria en el aula. Para ello, hemos atendido a la dimensión conceptual, la metodológica y la actitudinal requeridas para pasar a prácticas basadas en la indagación (Hinojosa y Sanmartí 2019). De manera que permitiese, no solo la aplicación de pasos metodológicos, sino también, la asunción de la complejidad de los fenómenos socioambientales cuyo abordaje requiere de la conjunción de varias perspectivas (Brocos y Jiménez Aleixandre, 2020) y de una mirada crítica, reflexiva y colectiva. La elección de la problemática a investigar desde el punto de vista didáctico - la implementación de ganadería y agricultura intensiva en grandes extensiones rurales-, aúna las características para ello: es un tema actual, próximo a la realidad del aprendiz (conocimiento cotidiano), representando, en sí, un sistema complejo que permite analizarlo desde varios ángulos y con relaciones temáticas muy potentes. Prueba de ello, es la amplia trama de problemas que hemos identificado que se prestan a ser objeto de investigación como contenido curricular y que favorece trabajar con el alumnado dicha complejidad. Así, se han desarrollado investigaciones sobre la salud, la dieta, la ecología, el modelo socioeconómico, el uso de los recursos, las características biológicas de los animales, del territorio o las prácticas ganaderas. Este planteamiento encaja en lo que Kelly (2016) denomina una purposeful activity situando al futuro profesorado en un ambiente de aprendizaje bajo la construcción social del conocimiento científico, en lugar de ofrecer una mera actividad de tipo experimental (la actividad de indagación tiene un fin último: se debe tomar una decisión fundamentada).

La primera fase de la actividad, organizar el contenido que advierten de la situación problema en un mapa/trama, nos da la evolución que experimenta su visión sobre dicha problemática y la capacidad de establecer relaciones (Hinojosa y Sanmartí 2019). No obstante, advertimos dificultad para transitar de relaciones más evidentes -como puede ser identificar las diferentes perspectivas o 
sectores implicados en la problemática- a relaciones más complejas, de dependencia, de influencia, de retroalimentaciones, etc.

Del análisis de los informes de investigación se han identificado diferentes procesos de trabajo desde el inicial al avanzado, resultando, principalmente, un adecuado desarrollo. No obstante, es interesante la caracterización de ciertas dificultades de aprendizaje relacionadas, por un lado, con la identificación de variables y, por otro lado, la formulación de hipótesis y la coherencia con la problemática advertida. Ambas dificultades, se retroalimentan negativamente. En cuanto al diseño metodológico, aunque se dan trabajos de campo (análisis del suelo, entrevistas), priman las planificaciones en base a consultas bibliográficas, fundamentalmente búsqueda de información en Internet (Solís y López-Lozano, 2015). Esto podría ser fruto del bagaje educativo tradicional del alumnado, deficiente en actividades experimentales. Además, de la tendencia en considerar que la documentación es una técnica previa y complementaria a la planificación del diseño experimental y no el único recurso.

En relación a las ideas conclusivas y toma de decisiones, la argumentación se enriquece dando paso a la interpretación de sus hallazgos y consultas, aunque también se aprecia, minoritariamente, una tendencia a la descripción frente a la interpretación. En el posicionamiento, prevalece la perspectiva ambiental y ecológica frente a un tímido apoyo inicial desde la perspectiva económica, dando valor a un desarrollo sostenible, posicionándose en contra de estas prácticas. No obstante, como ocurre en el caso del estudio de Brocos y Jiménez Aleixandre (2020) para argumentar sobre la idoneidad de tipos de dieta desde la visión ambiental, en nuestro caso, para su decisión, apelan a diversas cuestiones como el uso sostenible de los recursos, del territorio, la calidad del puesto de trabajo, el bienestar del animal y reflexiones acerca de hábitos de consumo y tipo de dieta, por ejemplo.

Respecto a la dimensión actitudinal, los resultados obtenidos sugieren que este tipo de secuenciación didáctica contextualizada en problemas cotidianos, facilita la sensibilización y toma de conciencia ante actividades con impacto socioambiental para lo cual la ciencia cobra relevancia (Aragón, 2017; Barraza y Castaño, 2012; Brocos y Jiménez Aleixandre, 2020; García-Díaz, 2001; Rivero y Solís, 2008).

Asimismo, el trabajo presenta continuidad tanto formativa como investigativa. Se presta a ampliar el diseño de la secuencia formativa incluyendo la dimensión de actuación en la competencia científica (Pigrau y Sanmartí, 2013) que promueva cambios de conducta y de mentalidad frente a una situación de emergencia climática. También, puede ser el inicio y foco central (como temática a enseñar) del posterior diseño de propuestas didácticas para alumnado de Primaria de los futuros docentes en el marco de la misma asignatura (Rivero et al., 2017) aunando la problematización del contenido desde una perspectiva ecosocial y la metodología investigativa.

\section{Referencias}

Acevedo, J. A., Vázquez, Á., Martín, M., Oliva, J. M., Acevedo, P., Paixão, F. y Manassero, A. (2005). Naturaleza de la ciencia y Educación científica para la participación ciudadana. Una revisión crítica. Revista Eureka sobre Enseñanza y Divulgación de las Ciencias, 2(2), 121-140.

Aragón Méndez, M $M^{\mathrm{a}}$ del M. (2017). Hacia una visión holística de la naturaleza de la ciencia en la formación inicial del profesorado de ciencia. Enseñanza de las ciencias: revista de investigación y experiencias didácticas, $\mathrm{N}^{\mathrm{o}}$ Extra 0, 3617-3622. Recuperado de https://www.raco.cat/index.php/Ensenanza/article/view/337120

Ballenilla, F. (1989). Los juegos de simulación de sistemas: un recurso didáctico necesario. Investigación en la Escuela, 8, 63-71.

Barraza, L. y Castaño, C. (2012) ¿Puede la enseñanza de la ciencia ayudar a construir una sociedad sostenible? Profesorado. Revista de Currículum y Formación de Profesorado, 16(2), 45-58. Recuperado de http://www.ugr.es/ recfpro/rev162ART3.pdf 
Bodmer, W. F. (1985). (Coord.). The public understanding of science. Londres: The Royal Society. Bravo Torija, B. y Jiménez Aleixandre, M. P. (2013). ¿Criaríamos leones en granjas? Uso de pruebas y conocimiento conceptual en un problema de acuicultura. Revista Eureka sobre Enseñanza y Divulgación de las Ciencias, 10(2), 122-135. Recuperado de http://hdl.handle.net/10498/15111

Brocos, P. y Jiménez Aleixandre, M. P. (2020). El impacto ambiental de la alimentación: argumentos de alumnado de Magisterio y Secundaria. Enseñanza de las Ciencias, 38(1), 127-145. DOI: $10.5565 / \mathrm{rev} / \mathrm{ensciencias} .2802$

Cañal, P., y Rivero, A. (1999). Cómo desarrollar una pequeña investigación científica. Sevilla: Material de aula, Universidad de Sevilla.

Caselles, A. (2008). Modelización y simulación de sistemas complejos. Valencia: Publicacions de la Universitat de València. Recuperado de https://www.uv.es/caselles/Mod1.pdf

Creswell, J.W. (2013). Qualitative Inquiry \& Research Design: Choosing Among the Five Approaches. Thousand Oaks, CA: SAGE Publications, Inc.

Cumbre Mundial del Clima Chile, COP25 (2019). Report of the Secretary-General on the 2019 Climate Action Summit and the Way Forward in 2020. Recuperado de https://www.un.org/es/climatechange/assets/pdf/cas_report_11_dec.pdf

Escrivá-Colomar, I. y Rivero, A. (2017). Progresión de las ideas de los futuros maestros sobre la construcción del conocimiento científico a través de mapas generados en una secuencia de actividades. Revista Eureka sobre Enseñanza y Divulgación de las Ciencias, 14(1), 199-214. Recuperado de http://hdl.handle.net/10498/18856

España, E. y Prieto, T. (2010). Problemas socio-científicos y enseñanza-aprendizaje de las ciencias. Investigación en la escuela, 71, 17-24.

García Barros, S. (2016). Conocimiento científico conocimiento didáctico: una tensión permanente en la formación docente. Campo Abierto, 35(1), 31-44.

García-Carmona, A., Vázquez Alonso, Á., y Manassero, M.A. (2011). Estado actual y perspectivas de la enseñanza de la naturaleza de la ciencia: una revisión de las creencias y obstáculos del profesorado. Enseñanza de las ciencias: revista de investigación y experiencias didácticas, 29(3), 403-412.Recuperado de https://www.raco.cat/index.php/Ensenanza/article/view/247899

García-Carmona, A., Vázquez Alonso, A. y Manassero, M.A. (2012). Comprensión de los estudiantes sobre naturaleza de la ciencia: análisis del estado actual de la cuestión y perspectivas. Enseñanza de las ciencias: revista de investigación y experiencias didácticas, 30(1), 23-34. Recuperado de https://www.raco.cat/index.php/Ensenanza/article/view/252558

García-Díaz, E. (2001). De los problemas científico a los problemas socioambientales (y vuelta). Alambique, 29, 25-33.

García-Díaz, E., Porlán, R., y Navarro, E. (2017). Los fines y los contenidos de enseñanza. En R. Porlán (Coord.), Enseñanza universitaria. Cómo mejorarla (pp. 55-72). Universidad de Sevilla.

García, F. (2000) Los modelos didácticos como instrumento de análisis y de intervención en la realidad educativa. Biblio $3 W$. Revista Bibliográfica de Geografía y Ciencias Sociales, V (205). Disponible en: http://www.ub.es/geocrit/b3w-207.htm

Harlen, W. (2015). Working with Big Ideas of Science Education. United Kingdon: ASE (Association for Science Education). Recuperado de http://www.ase.org.uk/resources/big-ideas/

Hinojosa, J. y Sanmartí, N. (2019). Indagando en el aula de ciencias: cómo progresan los métodos empleados por los docentes. Un estudio de caso. Investigación en la escuela, 99, 15-31. DOI: 10.12795/IE.2019.199.02

Kelly G. J. (2016) Methodological considerations for interactional perspectives on epistemic cognition. En J. A. Greene, W. A. Sandoval e I. Bråten (Eds.), Handbook of Epistemic Cognition (pp. 393-408). Nueva York: Routledge.

Kraser, R. B., y Hernández, S. A. (2020). Colorantes alimentarios y su relación con la salud: ¿cómo abordar esta problemática desde el estudio de las disoluciones? Revista Eureka sobre 
Enseñanza $y$ Divulgación de las Ciencias, 17(1), 1202. DOI: 10.25267/Rev_Eureka_ensen_divulg_cienc.2020.v17.i1.1202

Lederman N. G. y Lederman J.S. (2014). Research of Teaching and Learning of Nature of Science. En Norman G. Lederman and Sandra K. Abell (coord.), Handbook of Research on Science Education (pp. 600-620). Nueva York: Routledge.

López-Lozano, L. (2020). ¿Qué debemos saber sobre Ciencia?: un ciclo de mejora en la formación científica de los futuros docentes de Primaria. En R. Porlán y E. Navarro (Coords.), Ciclos de mejora en el aula. Año 2019. Experiencias de innovación docente (pp. 1328- 1348). Sevilla: Universidad de Sevilla. DOI: 10.12795/9788447221912

Manassero Mas, M.A., y Vázquez Alonso, Á. (2017). Enseñando la naturaleza del conocimiento científico mediante juegos. Enseñanza de las ciencias: revista de investigación y experiencias didácticas, $\quad \mathrm{N}^{\mathrm{o}} \quad$ Extra $\quad 0, \quad 367-374 . \quad$ Recuperado de https://www.raco.cat/index.php/Ensenanza/article/view/334281

Mengascini A., y Menegaz A. (2005). "El juego de las mariposas" propuesta didáctica para el tratamiento del cambio biológico. Revista Eureka sobre Enseñanza y Divulgación de las Ciencias, 2(3), 403-415. DOI: 10.25267/Rev_Eureka_ensen_divulg_cienc.2005.v2.i3.08

Moreno-Crespo, P., y Moreno-Fernández, O. (2015). Problemas socioambientales: concepciones del profesorado en formación inicial. Andamios, 12(29), 73-96.

Morin, E. (1995). Introducción al pensamiento complejo. Barcelona: Gedisa.

Morón Monge H., Morón Monge M.C., y Abril López, D. (2020). La rúbrica como instrumento para evaluar mapas conceptuales desde la construcción creativa de los conocimientos: Una propuesta participativa. Profesorado. Revista de Currículum y Formación de Profesorado, 24(1), 246-264. DOI: 10.30827/profesorado. v24i1.8514

Pigrau T., y Sanmartí N. (2013). La competencia científica. Rúbrica. En Tresor de recursos. Evaluar para aprender. Recuperado de https://www.tresorderecursos.com/copia-de-2-rubrica-comp-cc

Pujol, R.M. (2003). Didáctica de las Ciencias en la Educación Primaria. Síntesis: Madrid.

Rivero, A., y Solís, E. (2008). Promover la cultura científica para intervenir en el mundo. Cuadernos de Pedagogía, 384, 53- 56.

Rivero, A., y Wamba A. (2011). Naturaleza de la ciencia y construcción del conocimiento científico. La naturaleza de la ciencia como objetivo de enseñanza. En P. Cañal (coord.) Biología y Geología. Complementos de formación disciplinar (pp. 9-30). Barcelona: Graó.

Rivero, A., Martín Del Pozo, R., Solís, E., y Porlán, R. (2017). Didáctica de las ciencias para maestros. Madrid: Síntesis.

Rodríguez-Marín, F., Fernández-Arroyo, J. y García, E. (2014). Las hipótesis de transición como herramienta didáctica para la Educación Ambiental. Enseñanza de las ciencias: revista de investigación y experiencias didácticas, 32(3), 303-318.

Solís, E., y López-Lozano, L. (2015). Preocupaciones «energéticas» de los estudiantes de Magisterio. Alambique, 79, 49-57.

Solís, E., Porlán, R., Rivero, A., y Martín del Pozo, R. (2012). Las concepciones de los profesores de ciencias de secundaria en formación inicial sobre metodología de enseñanza. Revista Española de Pedagogía, 253, 495-514.

Solís, E., Luna, M. y Soler, M (1999). Los juegos de simulación una estrategia en la formación del profesorado. Perspectiva CEP. Revista de los Centros del Profesorado de Andalucía, 1, 121 131.

Vázquez, B., Jiménez, R., y Mellado, V. (2007). El desarrollo profesional del profesorado de ciencias como integración de la reflexión y la Práctica. La hipótesis de la complejidad. Revista Eureka sobre Enseñanza y Divulgación de las Ciencias, 4(3), 372-393.

Vilches, A., y Gil Pérez, D. (2012). La educación para la sostenibilidad en la universidad: el reto de la formación del profesorado. Profesorado. Revista de curriculum y formación del profesorado, 16(2), 25-43 
Ziman, J. (1986). Introducción al estudio de las ciencias. Barcelona: Ariel

CÓMO CITAR ESTE ARTÍCULO

López-Lozano, L., Rodríguez-Marín, F. y Solís Ramírez, E. (2021). Conocimiento científico, problemas socioambientales y formación inicial del Profesorado de Primaria. Didáctica de las Ciencias Experimentales y Sociales, 41, 171-188. DOI: 10.7203/DCES.41. 20564 